\title{
Identificación de las variantes genéticas asociadas al color de ojos verde y a la presencia de anillo peripupilar en humanos
}

\author{
Ángela Collado-Miralles \\ al262282@uji.es \\ Bárbara Hernando \\ hernandb@uji.es \\ Conrado Martínez-Cadenas \\ ccadenas@uji.es
}

\section{Resumen}

Introducción: El color de los ojos, rasgo hereditario poligénico, es una de las características físicas más visibles y diferenciables entre humanos. Por ello, la predicción del color del iris es ampliamente utilizada en medicina forense. Variaciones en genes involucrados en la pigmentación han sido asociadas a la variación en la pigmentación del iris entre individuos, especialmente para discernir entre ojos azules y marrones. Sin embargo, poco se conoce acerca de los determinantes genéticos que definen el color de ojos verde, así como el anillo peripupilar.

Objetivos: Analizar las variaciones genéticas que definen los patrones de pigmentación en el iris.

Metodología: Se obtuvo una muestra de ADN de 829 voluntarios para determinar el genotipo de 6 genes involucrados en pigmentación humana. La asociación entre color de ojos y genotipo se analizó mediante regresión logística. Resultados: Se confirma que el SNP rs12913832 localizado en HERC2/OCA2 es el mayor determinante de la variación en el color de ojos $(p<0,001)$. Aunque el alelo derivado C de rs12913832 se asocia al color de ojos azul, la mayoría de individuos con ojos verdes son portadores de dicho alelo. Polimorfismos en IRF4 y OCA2 también parecen influir en tener el iris verde. También observamos una asociación entre la presencia de anillo peripupilar y variaciones en el gen $S L C 24 A 4$, aunque únicamente en individuos heterocigotos para rs12896399. Conclusión: Las herramientas de predicción de características fenotípicas como el color de ojos deberían analizar los modelos de herencia, ya que el número de copias del alelo derivado parece influir en el patrón de pigmentación del iris.

Palabras clave: pigmentación humana, polimorfismos genéticos, asociación genética, color de ojos, anillo peripupilar. 


\begin{abstract}
Introduction: The colour of eyes, a polygenic hereditary trait, is one of the most visible and distinguishable physical characteristics among humans. For that reason, the prediction of iris colour is widely used in forensic medicine. Genetic variations in pigmentation-related genes have been associated with the variation of iris pigmentation between individuals, particularly to distinguish between blue and brown eyes. However, little is known about the genetic determinants responsible for the green colour, as well as the presence of pigmented ring on the iris.

Objectives: To analyse the genetic variations that determine the patterns of iris pigmentation.

Methodology: DNA samples from 829 volunteers were obtained in order to analyse the genotype of 6 genes involved on human pigmentation. The association between eye colour and genotype was analysed using logistic regression.

Results: Our results confirmed that the SNP rs12913832 located on $H E R C 2 / O C A 2$ is the major determinant of the natural eye colour variation $(p<0.001)$. Although the derived $C$ allele of rs 12913832 is associated with blue eye colour, most individuals with green eyes are carriers of that allele. Polymorphisms in IRF4 and OCA2 also seem to influence on having green iris. We also observed an association between the presence of pigmented ring on the iris and genetic variations in SLC24A4, although only in individuals heterozygous for rs12896399.

Conclusion: Prediction tools for phenotypic characteristics such as eye colour should take into account inheritance models, since the copy number of the derived allele carried seems to influence the pigmentation pattern of the iris.
\end{abstract}

Keywords: human pigmentation, genetic polymorphisms, genetic association, eye colour, pigmented ring.

\title{
Introducción
}

El color de los ojos es una de las características físicas más visibles y diferenciables entre humanos. El color del iris es un fenotipo altamente polimórfico, sobre todo en poblaciones de origen europeo, que está bajo un fuerte control genético (Deng y Xu 2017).

La pigmentación del iris está determinada por la cantidad, la distribución y el tipo de melanina presente en la capa anterior del estroma del iris (véase la figura 1). Podemos encontrar un gradiente de diferentes colores de ojos en la naturaleza, aunque esta variabilidad, en casi todos los casos, se puede reducir inequívocamente a tres amplias categorías cualitativas percibidas naturalmente por el ojo humano: azul/gris, verde/avellana (también llamado intermedio) y marrón/negro (Martínez-Cadenas, Peña-Chilet, Ibarrola-Villava y Ribas 2013). Los iris marrones tienen grandes cantidades de melanina y numerosos melanosomas que absorben la luz, de ahí el color oscuro mostrado. Aunque los ojos azules tienen un número similar de melanocitos que los ojos de color marrón, estos tienen un menor contenido de melanina en los melanosomas, de forma que la luz puede penetrar en el estroma, siendo parte de ella absorbida en la parte interna de los ojos, mientras que la demás es reflejada, dando apariencia de color azul. Los ojos verdes y de color miel tienen cantidades intermedias de melanina. 
Asimismo, la melanina no siempre se distribuye de forma uniforme por el estroma del iris, dando lugar a diferentes patrones de pigmentación en el iris. Uno de los ejemplos más claros es el anillo peripupilar, patrón de pigmentación que se caracteriza por presentar una zona circular de pigmentación alrededor de la pupila más oscura que el color basal del iris (Mackey, Wilkinson, Kearns y Hewitt 2011). Otros patrones de pigmentación no homogénea en el iris son, por ejemplo, la heterocromía completa y la heterocromía parcial.

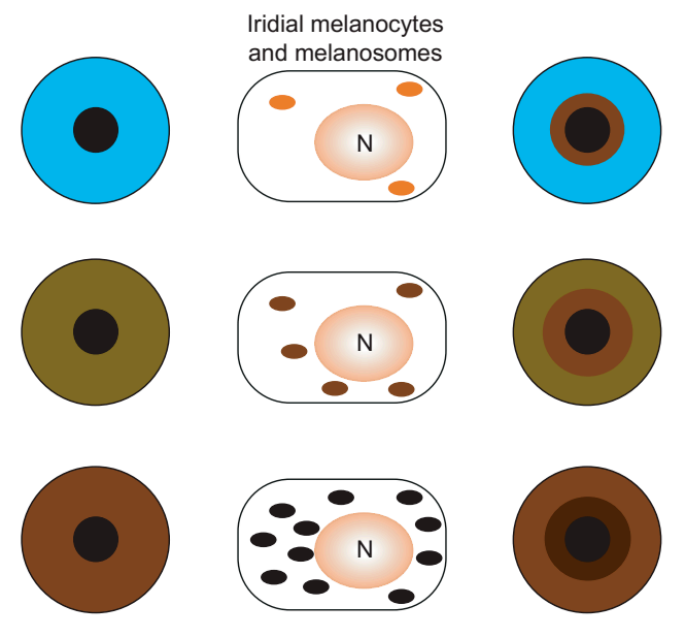

Figura 1. Distribución de la melanina en el iris de diferentes colores (Sturm y Frudakis 2004).

El color de los ojos es un rasgo fenotípico que sigue un complejo modelo de herencia poligénica, es decir, está principalmente determinado por factores genéticos con efectos relativamente pequeños que actúan juntos de forma cuantitativa, dando lugar a una amplia gama de colores entre el azul y el marrón oscuro, generados por interacciones genéticas complejas. En los últimos años, seis variantes genéticas localizadas en genes relacionados con la ruta de la síntesis de melanina se han identificado como los principales determinantes genéticos del color de ojos: rs12913832 en el gen HERC2, rs1800407 en OCA2, rs12896399 en SLC24A4, rs16891982 en SLC45A2, rs1393350 en TYR y rs12203592 en IRF4 (García-Borrón, Sánchez-Laorden y Jiménez-Cervantes 2005). Entre ellos, el polimorfismo rs12913832 localizado en el gen HERC2 (situado al lado del gen OCA2 en el genoma humano) ha sido descrito como el mayor determinante genético para el color de ojos, ya que parece explicar el $75 \%$ de la variación del color de ojos entre individuos (Sulem et al. 2007). Esta variante genética parece influir en la expresión del gen OCA2, el cual codifica para una proteína involucrada en la síntesis de melanina y la maduración de los melanosomas dentro del melanocito.

El estudio de los genes de la pigmentación humana puede ser de gran utilidad en diferentes campos, como en el campo de la medicina legal y forense. Por ejemplo, si se encuentra una muestra biológica sin identificar en una escena de un crimen o en una catástrofe natural, el análisis de variantes genéticas asociadas a rasgos fenotípicos de pigmentación facilitará la descripción fenotípica del individuo del cual pertenece la muestra biológica encontrada y, por lo tanto, su identificación (MartínezCadenas, Peña-Chilet, Ibarrola-Villava y Ribas 2013). Presentado este caso práctico, se ejemplifica la gran utilidad práctica que tiene la investigación de marcadores genéticos de rasgos físicos en la medicina forense. 
Debido a que la pigmentación del iris es un rasgo altamente determinado genéticamente, la predicción del color del iris a partir de una muestra biológica es una herramienta de gran interés y ampliamente aplicada en la genética forense (MartínezCadenas, Peña-Chilet, Ibarrola-Villava y Ribas 2013). Muestra de esta utilidad y aplicación práctica es la herramienta Irisplex, una herramienta robusta diseñada para predecir el color de ojos a partir de una muestra de ADN mediante la genotipación de únicamente las seis variantes genéticas que determinan en mayor medida la pigmentación del iris. Así pues, la herramienta Irisplex es aplicada en la genética forense para determinar si los ojos de los individuos de los cuales pertenece la muestra biológica son azules o marrones con una precisión del $90 \%$, siendo el resto de patrones de pigmentación clasificados como de color indefinido (categoría que incluye el color verde, miel o una mezcla de colores) (Walsh et al. 2011).

Así pues, Irisplex presenta deficiencias en la predicción del color de ojos verde (ya que es clasificado por descarte) y en la predicción de la presencia de patrones de pigmentación no uniformes en el iris, como es el anillo peripupilar. En ojos de color claro (azul/gris y verdes), la presencia de anillo peripupilar alrededor de la pupila es una característica apreciable a simple vista sin necesidad de utilizar una lámpara de hendidura (herramienta básica de oftalmología que permite ver en gran aumento las estructuras oculares). Por ello, puede ser útil predecir la presencia de anillo peripupilar para la diferenciación más precisa entre individuos. De ahí nace el interés por conocer más exhaustivamente las determinantes genéticas que definen que un individuo tenga un patrón de pigmentación en el iris concreto. La inclusión de dicha información en el sistema actual de predicción del color de ojos de un individuo puede ser de gran utilidad en el campo de la genética forense, puesto que implicaría una predicción más exacta de los rasgos de pigmentación del iris del individuo al cual pertenece la muestra de ADN encontrada.

Hasta el momento, poco se conoce de los determinantes genéticos que definen la presencia de anillo peripupilar, así como el color de ojos verde. Por ello, este estudio intentará esclarecer cuáles son las variantes genéticas que determinan la pigmentación del iris, especialmente el color verde y la presencia de anillo peripupilar (dos patrones de pigmentación del iris que han sido poco estudiados anteriormente).

\section{Métodos}

\section{Selección de la muestra y recogida de datos}

En este estudio observacional se incluyeron 829 individuos voluntarios (280 con ojos marrones, 275 con ojos verdes y 274 con ojos azules). Los individuos se reclutaron de forma consecutiva con una selección dirigida con el objetivo de conseguir un número equiparado de individuos con ojos azules/grises, verdes y marrones/negros para realizar un estudio de caso-control.

Para el análisis de asociación de anillo peripupilar se utilizaron 159 voluntarios con ojos claros (aquellos que habían contestado la pregunta de si tenían o no anillo peripupilar en el iris), de los cuales 39 presentaban anillo peripupilar. El resto de individuos (120) fueron utilizados como controles.

Los voluntarios incluidos en el estudio fueron captados en la Universitat Jaume I (estudiantes y trabajadores) y en varios hospitales de las provincias de Castellón, Valencia y Madrid (pacientes de los servicios de dermatología de dichos hospitales). Este estudio se engloba dentro de un proyecto de investigación el cual ha sido 
aprobado por la Comisión Deontológica de la Universitat Jaume I de Castellón. Todos los participantes firmaron un consentimiento informado.

Se utilizó un cuestionario estandarizado para recoger datos de filiación (edad, sexo y lugar de nacimiento), hábitos de exposición (uso de crema solar e historia de exposición solar), características de sensibilidad solar (capacidad de bronceado, número de nevus y presencia de lentigos solares o efélides) y características de pigmentación (color de ojos, pelo y piel).

De cada uno de los participantes se obtuvo una muestra de saliva mediante una torunda estéril (método no invasivo) para la posterior extracción del ADN genómico. La extracción del ADN se realizó utilizando QIAamp DNA Mini Kit (Qiagen, Hilden, Alemania), siguiendo el protocolo recomendado por el fabricante.

\section{Genotipado de variantes genéticas}

Se analizaron un total de 6 variantes localizadas en genes previamente asociados a rasgos de pigmentación humana: HERC2, OCA2, TYR, SLC45A2, IRF4 y SLC24A4. Todas las reacciones de genotipado se realizaron utilizando KASP SNP Genotyping Chemistry (LGC, Hoddesdon, Reino Unido) y TaqMan SNP Genotyping Assays (Applied Biosystems, California, EE. UU.). El análisis de la genotipación se llevó a cabo utilizando el equipo de PCR a tiempo real y utilizando el software SDS 2.1 para el análisis de discriminación alélica. Como medida de control de calidad, se incluyeron tres controles positivos (un heterocigoto, un homocigoto del alelo ancestral y un homocigoto del alelo derivado) y un control negativo por placa de 96 pocillos.

\section{Análisis estadístico}

Se realizó una regresión logística binaria para determinar si existe asociación entre el color de ojos y cada una de las variantes genéticas analizadas en el estudio, teniendo en cuenta el genotipo de cada individuo. Para cada uno de estos análisis se estimó el riesgo asociado a ser homocigoto del alelo ancestral, homocigoto del alelo derivado o heterocigoto de cada uno de los SNP. La asociación entre el genotipo y la pigmentación del iris se expresa como odd ratio (OR) y su intervalo de confianza (IC $95 \%$ ). Se obtuvo el valor $\mathrm{p}$ asociado a cada uno de los análisis de asociación realizados.

En todos los análisis llevados a cabo en el estudio se limitó un valor p menor de 0,05 para considerar los resultados como estadísticamente significativos. Los análisis estadísticos se realizaron con el software SPSS v25.

\section{Resultados}

Este estudio se centra en realizar un análisis para conocer los determinantes genéticos del patrón de pigmentación del iris, especialmente del color verde de ojos y la presencia de anillo peripupilar. Con este objetivo en mente, se analizó el genotipo de 6 variantes genéticas asociadas a la variación natural del color de ojos en 829 individuos voluntarios.

De los voluntarios incluidos en el estudio, 275 tenían los ojos verdes. El genotipo de dichos individuos se comparó con los individuos que tenían los ojos azules y marrones. Como se puede observar en la tabla 1, existen diversas variantes genéticas 
que parecen influir significativamente con tener el iris de color verde $(O R>1$, $\mathrm{p}<0,05)$.

En primer lugar, el polimorfismo rs12913832 en HERC2 parece ser el mayor determinante del color verde de ojos. Según nuestros resultados, los individuos con los ojos verdes tienden a ser heterocigotos para esta variante genética (CT), teniendo 3,13 veces más riesgo relativo de tener los ojos verdes que los individuos homocigotos para el alelo de referencia $\left(p=1,78 \times 10^{-8}\right)$.

Tabla 1

Asociación genética de los polimorfismos estudiados con el color de ojos verde

\begin{tabular}{|c|c|c|c|c|c|c|c|c|}
\hline \multirow[b]{2}{*}{ Gen } & \multirow[b]{2}{*}{ SNP } & \multirow[b]{2}{*}{ Genotipo } & \multicolumn{2}{|c|}{ Ojos verdes } & \multicolumn{2}{|c|}{ Ojos no verdes } & \multirow[b]{2}{*}{ OR (95\% IC) } & \multirow[b]{2}{*}{ valor $p$} \\
\hline & & & $\mathbf{N}$ & $\%$ & $\mathbf{N}$ & $\%$ & & \\
\hline & & $\mathrm{CC}$ & 165 & 61,3 & 372 & 66,5 & 1,0 & Referencia \\
\hline \multirow[t]{3}{*}{ IRF4 } & rs12203592 & $\mathrm{CT}$ & 86 & 32,0 & 169 & 30,2 & $1,15(0,84-1,58)$ & 0,397 \\
\hline & & TT & 18 & 6,7 & 18 & 3,2 & $2,26(1,14-4,44)$ & 0,019 \\
\hline & & TT & 42 & 15,7 & 147 & 26,8 & 1,0 & Referencia \\
\hline \multirow[t]{3}{*}{ HERC2 } & rs12913832 & $\mathrm{CT}$ & 180 & 67,2 & 201 & 36,6 & $3,13(2,11-4,66)$ & $1,78 E-8$ \\
\hline & & $\mathrm{CC}$ & 46 & 17,2 & 201 & 36,6 & $0,80(0,50-1,28)$ & 0,354 \\
\hline & & $\mathrm{CC}$ & 198 & 73,6 & 464 & 84,1 & 1,0 & Referencia \\
\hline \multirow[t]{2}{*}{ OCA2 } & rs1800407 & CT & 62 & 23,0 & 86 & 15,6 & $1,69(1,17-2,44)$ & 0,005 \\
\hline & & TT & 9 & 3,4 & 2 & 0,3 & $10,55(2,26-49,25)$ & 0,003 \\
\hline \multirow{4}{*}{ TYR } & & GG & 137 & 51,3 & 325 & 58,7 & 1,0 & Referencia \\
\hline & rs1393350 & $A G$ & 107 & 40,1 & 200 & 36,2 & $1,27(0,93-1,73)$ & 0,130 \\
\hline & & AA & 23 & 8,6 & 28 & 5,1 & $1,95(1,08-3,50)$ & 0,026 \\
\hline & & GG & 78 & 29,0 & 157 & 28,7 & 1,0 & Referencia \\
\hline \multirow[t]{3}{*}{ SLC24A4 } & rs12896399 & GT & 111 & 41,3 & 253 & 46,3 & $0,88(0,62-1,26)$ & 0,49 \\
\hline & & TT & 80 & 29,7 & 137 & 25,0 & $1,16(0,80-1,73)$ & 0,41 \\
\hline & & GG & 222 & 82,5 & 437 & 79,2 & 1,0 & Referencia \\
\hline \multirow[t]{2}{*}{ SLC45A2 } & rs16891982 & GC & 43 & 16,0 & 94 & 17,0 & $0,90(0,61-1,34)$ & 0,603 \\
\hline & & $\mathrm{CC}$ & 4 & 1,5 & 21 & 3,8 & $0,36(0,13-1,11)$ & 0,075 \\
\hline \multicolumn{9}{|c|}{$\begin{array}{l}\text { Abreviaturas: IC, intervalo de confianza }(95 \%) ; \mathrm{N} \text {, número de individuos del s } \\
\text { ratio; SNP, single nucleotide polymorphism } \\
\text { La negrita indica que el resultado es estadísticamente significativo }(p<0,05) \\
\text { El valor p ha sido obtenido mediante regresión logística binaria }\end{array}$} \\
\hline
\end{tabular}

Además, se puede observar que también existe una asociación significativa entre el color de ojos verde y la variante rs1800407 del gen OCA2, ya que tener una copia del alelo derivado $(T)$ se asocia con tener el color verde con una OR de 1,69 $(p=0,005)$. Sin embargo, poseer dos copias del alelo rs $1800407^{\star} \mathrm{T}$ incrementa considerablemente el riesgo de tener el color de ojos verde $(O R=10,55, p=0,003)$.

Por último, los resultados también indican que ser homocigoto tanto para el alelo derivado (A) del polimorfismo rs1393350 en TYR se asocia con tener los ojos de color verde $(O R=1,95, p=0,026)$. Asimismo, el color de ojos verde también se asocia de manera significativa con ser homocigoto para el alelo derivado $(T)$ del polimorfismo rs12203592 del gen IRF4 (OR = 2,26, $p=0,019)$.

Otro objetivo del trabajo era conocer los determinantes genéticos de la presencia del anillo peripupilar, ya que es un patrón de pigmentación del iris poco estudiado hasta el momento. Como se ha descrito en la introducción, el anillo peripupilar se define como una zona circular alrededor de la pupila con una pigmentación más oscura que el color basal del iris. Este rasgo se aprecia a simple vista en los ojos de color claro, sin necesidad de utilizar una lámpara de hendidura (herramienta básica de oftalmología 
que permite ver en gran aumento las estructuras oculares). Así pues, para analizar las variantes genéticas que determinan el anillo peripupilar, solo se incluyeron sujetos con ojos claros que habían respondido a la pregunta de si tenían o no anillo peripupilar en el iris, ya que en los ojos oscuros el anillo pasa desapercibido. De los 159 voluntarios incluidos en dicho análisis, 39 individuos presentaban anillo peripupilar.

Tal y como se observa en la tabla 2, nuestros resultados no indican ninguna asociación estadísticamente significativa entre la presencia del anillo peripupilar y el genotipo del individuo (ser homocigoto para el alelo ancestral, ser heterocigoto o ser homocigoto para el alelo derivado) para ninguna de las variantes genéticas estudiadas.

Tabla 2

Asociación genética de las variantes genéticas con la presencia de anillo peripupilar

\begin{tabular}{|c|c|c|c|c|c|c|c|c|}
\hline \multirow[b]{2}{*}{ Gen } & \multirow[b]{2}{*}{ SNP } & \multirow[b]{2}{*}{ Genotipo } & \multicolumn{2}{|c|}{$\begin{array}{c}\text { Anillo } \\
\text { peripupilar }\end{array}$} & \multicolumn{2}{|c|}{$\begin{array}{c}\text { No anillo } \\
\text { peripupilar }\end{array}$} & \multirow[b]{2}{*}{ OR (95 \% IC) } & \multirow[b]{2}{*}{ valor $p$} \\
\hline & & & $\mathbf{N}$ & $\%$ & $\mathbf{N}$ & $\%$ & & \\
\hline & & $\mathrm{CC}$ & 19 & 48,7 & 60 & 50,0 & 1,0 & Referencia \\
\hline \multirow[t]{2}{*}{ IRF4 } & rs12203592 & CT & 19 & 48,7 & 48 & 40,0 & $1,25(0,60-2,62)$ & 0,555 \\
\hline & & TT & 1 & 2,6 & 12 & 10,0 & $0,26(0,03-2,16)$ & 0,214 \\
\hline & & TT & 8 & 21,1 & 13 & 11,1 & 1,0 & Referencia \\
\hline \multirow[t]{3}{*}{ HERC2 } & rs12913832 & CT & 18 & 47,4 & 47 & 40,2 & $0,62(0,22-1,75)$ & 0,369 \\
\hline & & $\mathrm{CC}$ & 12 & 31,6 & 57 & 48,7 & $0,34(0,12-1,01)$ & 0,051 \\
\hline & & $\mathrm{CC}$ & 27 & 71,1 & 95 & 79,8 & 1,0 & Referencia \\
\hline \multirow[t]{2}{*}{ OCA2 } & rs1800407 & CT & 9 & 23,7 & 22 & 18,5 & $1,44(0,59-3,49)$ & 0,420 \\
\hline & & TT & 2 & 5,3 & 2 & 1,7 & $3,52(0,47-26,16)$ & 0,219 \\
\hline \multirow{4}{*}{$T Y R$} & & GG & 22 & 57,9 & 64 & 54,2 & 1,0 & Referencia \\
\hline & rs1393350 & $A G$ & 14 & 36,8 & 46 & 39,0 & $0,89(0,41-1,91)$ & 0,757 \\
\hline & & AA & 2 & 5,3 & 8 & 6,8 & $0,73(0,14-3,69)$ & 0,701 \\
\hline & & GG & 11 & 28,9 & 47 & 40,2 & 1,0 & Referencia \\
\hline \multirow[t]{2}{*}{ SLC24A4 } & rs12896399 & GT & 23 & 60,5 & 50 & 42,7 & $1,97(0,86-4,47)$ & 0,107 \\
\hline & & TT & 4 & 10,5 & 20 & 17,1 & $0,86(0,24-3,01)$ & 0,807 \\
\hline & & GG & 26 & 72,2 & 95 & 79,2 & 1,0 & Referencia \\
\hline \multirow[t]{2}{*}{$S L C 45 A 2$} & rs16891982 & GC & 9 & 25,0 & 19 & 15,8 & $1,73(0,70-4,27)$ & 0,234 \\
\hline & & $\mathrm{CC}$ & 1 & 2,8 & 6 & 5,0 & $0,61(0,07-5,29)$ & 0,653 \\
\hline \multicolumn{9}{|c|}{$\begin{array}{l}\text { Abreviaturas: IC, intervalo de confianza }(95 \%) ; \mathrm{N} \text {, número de individuos del subs } \\
\text { ratio; SNP, single nucleotide polymorphism } \\
\text { La negrita indica que el resultado es estadísticamente significativo }(p<0,05) \\
\text { El valor } \mathrm{p} \text { ha sido obtenido mediante regresión logística binaria }\end{array}$} \\
\hline
\end{tabular}

No obstante, cabe destacar que un $60,5 \%$ de los individuos con anillo peripupilar eran heterocigotos para la variante rs 12896399 en SLC24A4, mientras que únicamente un $42,7 \%$ de los individuos sin presencia de anillo peripupilar tenían dicho genotipo. Este hecho nos hizo pensar que esta variante genética seguía un modelo de herencia superdominante, según el cual los individuos heterocigotos presentan un rasgo fenotípico más acentuado que ambos homocigotos. Por este motivo, llevamos a cabo un análisis de asociación entre la variante rs12896399 en SLC24A4 y la presencia de anillo peripupilar en el iris, teniendo en cuenta el modelo de herencia superdominante $\mathrm{y}$, por lo tanto, obteniendo el OR asociado a ser heterocigoto frente a ser homocigoto de cualquiera de los dos posibles alelos de SLC24A4 rs12896399. Los resultados indican que existe una asociación estadísticamente significativa entre la presencia de anillo peripupilar y el ser heterocigoto (GT) para la variante genética rs12896399 del gen SLC24A4 (OR = 2,49 [1,09-5,66], $p=0,027)$, respecto a ser homocigoto. 


\section{Discusión y conclusiones}

Este estudio se centra en analizar el genotipo de 6 variantes genéticas, localizadas en genes relacionados con la pigmentación humana, específicamente con el color de ojos, en un total de 829 españoles con el objetivo de ampliar el conocimiento acerca de la genética de la pigmentación de iris, especialmente el color verde y la presencia de anillo peripupilar (dos patrones de pigmentación del iris que han sido poco estudiados anteriormente).

Nuestros resultados confirman estudios previos que definen el polimorfismo rs12913832 localizado en el gen HERC2 como el determinante genético que explica la mayoría de la variación natural del color de los ojos en europeos (Martínez-Cadenas, Peña-Chilet, Ibarrola-Villava y Ribas 2013; Eiberg et al. 2008) e indican que el resto de los polimorfismos aparentemente implicados en el color de ojos —rs1800407 en OCA2, rs12896399 en SLC24A4, rs16891982 en SLC45A2, rs1393350 en TYR y rs12203592 en IRF4 - tienen un papel relativamente menor (Gudbjartsson et al. 2008; Sulem et al. 2007; Han et al. 2008). Estos estudios previos de asociación genética indican que los individuos portadores del alelo derivado $\mathrm{C}$ del polimorfismo rs12913832 en HERC2 suelen tener los ojos de color azul, mientras que ser homocigoto del alelo ancestral T se asociaba a tener los ojos oscuros. Nuestro estudio indica que la mayoría de individuos con los ojos verdes son heterocigotos para HERC2 rs12913832 (CT), de forma que el riesgo relativo de tener los ojos verdes incrementa en 3,13 veces respecto a ser homocigoto del alelo ancestral. Asimismo, ser portador de los alelos derivados de las variantes rs1800407 en OCA2, rs1393350 en TYR y rs12203592 en IRF4 se asocia con tener los ojos de color verde.

En cuanto al anillo peripupilar, los resultados obtenidos en el análisis de asociación indican que no existe ninguna asociación estadísticamente significativa entre la presencia de anillo peripupilar y las variantes genéticas estudiadas según el genotipo de cada individuo. Sin embargo, la distribución de los individuos con anillo y sin anillo peripupilar según el genotipo de la variante rs12896399 en SLC24A4 nos hicieron sospechar que únicamente los individuos heterocigotos en dicha variante mostraban el fenotipo estudiado. Nuestras sospechas fueron confirmadas al realizar el análisis de asociación entre la presencia del anillo peripupilar y la variante rs12896399 teniendo en cuenta los diferentes modelos de herencia genética que se pueden encontrar en la naturaleza (codominante, dominante, recesivo y superdominante). De hecho, estos análisis indicaron que existe una asociación entre la presencia de anillo peripupilar y el ser heterocigoto para rs12896399 en el gen SLC24A4, según el modelo de herencia superdominante. El patrón de herencia superdominante se da cuando únicamente los individuos heterocigotos muestran el fenotipo, es decir, cuando es necesario ser portador de ambos alelos para expresar un fenotipo concreto. Nuestros resultados concuerdan con un estudio previo que correlaciona la variante rs4900109 del gen SLC24A4 con la presencia de un anillo de pigmentación más oscura alrededor de la pupila (Larsson et al. 2011). Según este estudio, esta variante genética puede influenciar sobre dónde se deposita la melanina en el iris en vez de en el color basal del ojo. Destacar que este polimorfismo genético está altamente ligado a la variante que nosotros analizamos en este estudio (rs12896399), es decir, ambas variantes se heredan en bloque. Esto indica que, aunque se analice una variante genética distinta a la estudiada por Larsson y sus colaboradores, los resultados de ambos estudios son equivalentes.

Como se ha comentado anteriormente, la necesidad de profundizar en el conocimiento de los determinantes genéticos que definen los patrones de pigmentación del iris recae en su gran utilidad en la genética forense. Así pues, los avances en este estudio pueden servir para mejorar los sistemas de predicción del color de ojos basados en la 
genética utilizados actualmente. Si analizamos el diseño de Irisplex, esta herramienta de predicción únicamente tiene en cuenta el efecto acumulativo del riesgo asociado a tener el alelo derivado, de forma que el modelo de herencia genética que se utiliza es el aditivo (Walsh et al. 2011). Es decir, cuando el individuo es heterocigoto para una variante genética, se le suma una vez el riesgo asociado al alelo derivado, mientras que cuando el individuo es homocigoto para el alelo derivado, el riesgo asociado se multiplica por dos. No obstante, existen otros modelos de herencia genética en la naturaleza (como el modelo codominante, dominante, recesivo y superdominante) que deberían tenerse en cuenta, ya que tener una o dos copias del alelo derivado parece influir en el patrón de pigmentación del iris entre individuos de forma no aditiva, tal y como muestran nuestros resultados en el caso del polimorfismo rs12913832 en HERC2 para los ojos verdes y del polimorfismo rs12896399 en SLC24A4 para la presencia de anillo peripupilar.

Irisplex es una herramienta ampliamente utilizada en medicina forense para, a partir de una muestra de ADN desconocida o anónima, clasificar los ojos de un individuo como azules o marrones con una precisión del $90 \%$. Sin embargo, aquellas muestras que no puedan ser clasificadas dentro de estas dos categorías (no cumplen los criterios establecidos para clasificarse como ojos azules o marrones), son incluidas en el grupo de color de ojos intermedio, donde se incluye el color verde. Así pues, Irisplex no ha sido diseñada para predecir específicamente a los individuos con ojos verdes o con anillo peripupilar. Por este motivo, es necesario actualizar la herramienta Irisplex para ser capaces de mejorar la identificación de individuos con ojos verdes o anillo peripupilar mediante la incorporación del conocimiento generado en los estudios de investigación relacionados con la genética de la pigmentación del iris.

\section{Referencias bibliográficas}

Deng, Lian y Shuhua Xu. 2017. "Adaptation of human skin color in various populations». Hereditas 155(1). http://doi.org/10.1186/s41065-017-0036-2

Eiberg, Hans, Jesper Troelsen, Mette Nielsen, Annemette Mikkelsen, Jonas MengelFrom, Klaus W. Kjaer y Lars Hansen. 2008. «Blue eye color in humans may be caused by a perfectly associated founder mutation in a regulatory element located within the HERC2 gene inhibiting OCA2 expression». Human Genetics 123(2): 177-187. http://doi.org/10.1007/s00439-007-0460-x

García-Borrón, José C., Berta Sanchez-Laorden y Celia Jiménez-Cervantes. 2005. «Melanocortin-1 receptor structure and functional regulation». Pigment Cell Research 18(6): 393-410. http://doi.org/10.1111/j.1600-0749.2005.00278.x

Gudbjartsson, Daniel F., Patrick Sulem, Simon N. Stacey, Alisa M. Goldstein, Thorunn Rafnar, Bádur Sigurgeirsson, Kristrun R. Benediktsdottir... y Kari Stefansson. 2008. "ASIP and TYR pigmentation variants associate with cutaneous melanoma and basal cell carcinoma». Nature Genetics 40(7): 886-891. http://doi.org/10.1038/ng.161

Han, Jiali, Peter Kraft, Hongmei Nan, Qun Guo, Constance Chen, Abrar Qureshi, Susan E. Hankinson, Frank B. Hu... y David J. Hunter. 2008. «A Genome-Wide Association Study Identifies Novel Alleles Associated with Hair Color and Skin Pigmentation». Plos Genetics 4(5): e1000074. http://doi.org/10.1371/journal.pgen.1000074

Larsson, Mats, David L. Duffy, Gu Zhu, Jimmy Z. Liu, Stuart Macgregor, Allan F. McRae, Margaret J. Wright, Richard A. Sturm... y Sarah E. Medland. 2011. «GWAS Findings for Human Iris Patterns: Associations with Variants in Genes that Influence Normal Neuronal Pattern Development». The American Journal of Human Genetics 89(2): 334-343. http://doi.org/10.1016/j.ajhg.2011.07.011 
Mackey, David A., Colleen H. Wilkinson, Lisa S. Kearns y Alex W. Hewitt. 2011. "Classification of iris colour: review and refinement of a classification schema». Clinical \& Experimental Ophthalmology 39(5): 462-471. http://doi.org/10.1111/j.1442-9071.2010.02487.x

Martínez-Cadenas, Conrado, María Peña-Chilet, Maider Ibarrola-Villava y Gloria Ribas. 2013. "Gender is a major factor explaining discrepancies in eye colour prediction based on HERC2/OCA2 genotype and the IrisPlex model». Forensic Science International: Genetics 7(4): 453-460. http://doi.org/10.1016/j.fsigen.2013.03.007

Sturm, Richard A. y Tony Frudakis. 2004. «Eye colour: portals into pigmentation genes and ancestry». Trends in Genetics 20: 327-332. http://doi.org/10.1016/j.tig.2004.06.010

Sulem, Patrick, Daniel Fannar Gudbjartsson, Simon N. Stacey, Agnar Helgason, Thorunn Rafnar, Kristinn Pétur Magnusson, Andrei Manolescu, Ari Karason... y Kari Stefansson. 2007. "Genetic determinants of hair, eye and skin pigmentation in Europeans». Nature Genetics 39(12): 1443-1452. http://doi.org/10.1038/ng.2007.13

Walsh, Susan, Fan Liu, Kaye Ballantyne, Mannis van Oven, Oscar Lao y Manfred Kayser. 2011. «IrisPlex: A sensitive DNA tool for accurate prediction of blue and brown eye colour in the absence of ancestry information». Forensic Science International: 